\title{
Soroprevalência e fatores de risco para a leptospirose em cães de Campina Grande, Paraíba
}

[Seroprevalence and risk factors for leptospirosis in dogs from Campina Grande, State of Paraiba, Brazil]

\author{
C.S.A. Batista ${ }^{1}$, C.J. Alves ${ }^{1}$, S.S. Azevedo ${ }^{2}$, S.A. Vasconcellos $^{2}$, Z.M. Morais ${ }^{2}$, I.J. Clementino ${ }^{1}$, \\ F.A.L. Alves ${ }^{1}$, F.S. Lima ${ }^{1}$, J.O. Araújo Neto ${ }^{1}$ \\ ${ }^{1}$ Centro de Saúde e Tecnologia Rural - Universidade Federal de Campina Grande - Patos, PB \\ ${ }^{2}$ Faculdade de Medicina Veterinária e Zootecnia - Universidade de São Paulo \\ Av. Prof. Dr. Orlando Marques de Paiva, 87 \\ 05508-000 - São Paulo, SP
}

\begin{abstract}
RESUMO
Investigou-se a prevalência de leptospirose em cães da cidade de Campina Grande, PB, e realizou-se um estudo de fatores de risco para a infecção. Foram examinadas 285 amostras de soro sangüíneo de cães colhidas durante a campanha de vacinação anti-rábica animal, conduzida em setembro de 2003. O diagnóstico da leptospirose foi realizado pela técnica de soroaglutinação microscópica, utilizando-se uma coleção de 22 sorovares. Para a caracterização do sorovar mais provável, levou-se em conta a titulação e a freqüência. A prevalência encontrada foi de 21,4\% (IC 95\% = 16,8\%-26,6\%), com maior freqüência dos sorovares autumnalis $(7,4 \%)$, copenhageni $(6 \%)$ e canicola $(2,1 \%)$. A análise de regressão logística multivariada mostrou que os fatores de risco para a leptospirose foram: idade superior a um ano (odds ratio $=3,00 ; P=0,006$ ), raça não definida (odds ratio $=4,02 ; P=0,011$ ) e ocorrência de enchentes (odds ratio $=2,32 ; P=0,039)$.
\end{abstract}

Palavras-chave: cão, prevalência, leptospirose, fator de risco

\begin{abstract}
The prevalence of leptospirosis was investigated in dogs from Campina Grande city, State of Paraiba, Brazil, and the risk factors for infection were analyzed. Two hundred and eighty five blood samples were collected from dogs during the rabies vaccination campaign, in September 2003. The diagnostic method run for leptospirosis was the microscopic agglutination test, using a batch of 22 leptospiral serovars. The most prevalent serovar was found crossing the results of frequency and titer of agglutinins. The prevalence was $21.4 \%(95 \% \mathrm{CI}=16.8 \%-26.6 \%)$ and most frequent reactant serovars were autumnalis (7.4\%), copenhageni (6\%) and canicola (2.1\%). The multivariate logistic regression analysis showed that the risk factors for leptospirosis were: age older than one year (odds ratio $=3.00 ; P=0.006$ ), mixed breed (odds ratio $=4.02 ; P=0.011$ ) and occurrence of floods in the area (odds ratio $=2.32 ; P=0.039$ ).
\end{abstract}

Keywords: dog, prevalence, leptospirosis, risk factor

Recebido para publicação em 19 de abril de 2004

Recebido para publicação, após modificações, em 4 de novembro de 2004

*Autor para correspondência (corresponding author)

E-mail: sergio@vps.fmvz.usp.br 


\section{Batista et al.}

\section{INTRODUÇÃO}

A leptospirose, doença bacteriana infectocontagiosa que acomete o homem e os animais domésticos e silvestres, amplamente disseminada, é de considerável importância como problema econômico e de saúde pública (Faine et al., 1999). A ocorrência de leptospirose é variável em diferentes partes do mundo, podendo-se observar tanto a forma esporádica quanto a endêmica. Os surtos se reproduzem por exposição à água contaminada com urina ou tecidos provenientes de animais infectados (Vasconcellos, 1993), particularmente nas ocasiões em que ocorrem elevados índices de precipitações pluviométricas, e nas regiões em que o solo apresenta reação neutra ou levemente alcalina, associando-se ainda a variedade de espécies hospedeiras que facilitam a cadeia de eventos necessários para a transmissão da doença.

Dentre as modalidades de fonte de infecção dos animais acometidos, da maior relevância é o papel dos portadores (convalescentes e sadios), excretores de leptospiras a quem se atribui a maior parcela de culpa pela persistência de focos da doença. Devido à longa duração dessa condição e à ampla facilidade de deslocamento, por não manifestar sinais de infecção, eles se tornam os reservatórios de manutenção do agente no ambiente (Vasconcellos, 1993). Entre os animais domésticos, em ambiente urbano, a principal fonte de infecção da leptospirose humana são os cães. Esses animais vivem em contato direto com os seres humanos e podem eliminar leptospiras vivas pela urina durante meses, mesmo sem apresentar nenhum sinal clínico (Faine et al., 1999).

Vários inquéritos sorológicos realizados em cães no Brasil retratam a variabilidade da distribuição de sorovares de Leptospira spp predominantes nas diferentes localidades. Furtado et al. (1997) examinaram 260 cães em Pelotas, RS, e encontraram prevalência de $28,9 \%$, com destaque para os sorovares canicola e icterohaemorrhagiae. Avila et al. (1998) encontraram $34,8 \%$ de reatores em 425 cães em Pelotas, RS, com predomínio dos sorovares canicola, icterohaemorrhagiae e copenhageni. Alves et al. (2000) encontraram 20\% de reatores em 114 cães da cidade de Patos, PB, com destaque para os sorovares autumnalis, butembo, grippotyphosa e australis. Jouglard e Brod (2000), em Pelotas, verificaram 2,7\% de positividade em 489 cães, com destaque para os sorovares icterohaemorrhagiae, australis, copenhageni, pyrogenes, sentot e canicola. Em Santana de Parnaíba, SP, Mascolli et al. (2002) examinaram 410 amostras de soro de cães e encontraram $15,0 \%$ de positividade, com destaque para os sorovares copenhageni, canicola e hardjo. Fávero et al. (2002) verificaram $17,9 \%(137 / 795)$ e $19,7 \%$ (37/187) de positividade, com predomínio dos sorovares copenhageni e icterohaemorrhagiae e apenas do sorovar pyrogenes, em cães dos estados de São Paulo e Piauí, respectivamente.

Os objetivos deste trabalho foram determinar a prevalência de leptospirose em cães de Campina Grande, e verificar os possíveis fatores de risco para a doença.

\section{MATERIAL E MÉTODOS}

O experimento foi conduzido durante a campanha de vacinação anti-rábica animal do município de Campina Grande, PB, realizada em setembro de 2003, onde foi viabilizada a utilização dos postos fixos de vacinação como locais de colheita de sangue dos cães, para a obtenção de uma amostra que representasse a realidade dos vários bairros. Para tanto, a colheita acompanhou o cronograma utilizado na campanha de vacinação, que passa por todos os bairros da cidade.

A amostra colhida foi calculada com base na população total de cães da cidade, estimada a partir da população humana de 335.000 habitantes (Brasil, 2003). Para o cálculo da proporção cão/homem, foi utilizada a relação de 1:10 (Reichmann et al., 1999), que redundou em um total de 33.500 animais. O cálculo da amostra foi feito com o programa EpiInfo 6.0, considerando-se um nível de confiança de 95\%, prevalência esperada de $20 \%$ e erro estatístico de $5 \%$, resultando no $\mathrm{N}$ amostral de 244 . Por motivo de segurança, foram colhidas 285 amostras de sangue.

Em cada posto de vacinação, o número de amostras colhidas foi previamente determinado por sorteio probabilístico aleatório de acordo com a ordem cronológica, até perfazer o total de 
285 amostras. Assim, postos com maior número de animais tiveram maior número de amostras colhidas. A colheita foi realizada após a aplicação da vacina anti-rábica nos cães sorteados. Os proprietários dos cães responderam a um questionário, que serviu de parâmetro para a análise de fatores de risco. Foram investigadas questões como presença de roedores, contato com açude e ocorrência de enchentes, e características individuais, como sexo, raça, idade, tipo de manejo e vacinação. As informações dos questionários foram inseridas em um formulário eletrônico elaborado no programa de computador Microsoft Access ${ }^{\circledR}$.

O diagnóstico sorológico da leptospirose foi realizado pela técnica de soroaglutinação microscópica (Galton et al., 1965), com uma coleção de antígenos vivos que incluiu os sorovares: australis, bratislava, autumnalis, butembo, castellonis, bataviae, canicola, whitcombi, cynopteri, grippotyphosa, hebdomadis, icterohaemorrhagiae, panama, pomona, pyrogenes, hardjo, wolffi, shermani, tarassovi, andamana, patoc e sentot. Os soros foram triados na diluição de 1:100, e aqueles que apresentaram $50 \%$ ou mais de aglutinação foram titulados pelo exame de uma série de diluições geométricas de razão dois. O título do soro foi a recíproca da maior diluição que apresentou resultado positivo. Os antígenos eram examinados ao microscópio de campo escuro, previamente aos testes, a fim de verificar a mobilidade e a presença de auto-aglutinação ou de contaminantes. Para a caracterização do sorovar mais provável, considerou-se, para tanto, o sorovar que apresentou maior título e o maior número de animais caracterizados como positivos. Caso um animal reagisse para dois ou mais sorovares, ele era considerado positivo para o sorovar de maior título.

Para o estudo de fatores de risco, inicialmente foi realizada uma análise univariada pelo teste de qui-quadrado, e as variáveis que apresentaram $\mathrm{P}<0,20$ foram selecionadas e oferecidas para a regressão logística multivariada. Foi empregado o método incondicional da regressão logística (stepwise forward), conforme preconizado por Hosmer e Lemeshow (1989). Os cálculos foram feitos pelo programa SPSS for Windows versão 10.0. Para todas as análises, o nível de significância adotado foi de 0,05 .

\section{RESULTADOS E DISCUSSÃO}

Sessenta e uma amostras foram positivas pela técnica de soroaglutinação microscópica aplicada à leptospirose, obtendo-se prevalência de 21,4\% (IC $95 \%=16,8 \%-26,6 \%$ ). Prevalências superiores foram obtidas por Furtado et al. (1997) e por Ávila et al. (1998), em Pelotas, $28,9 \%$ e $34,8 \%$, respectivamente. No entanto, percentuais inferiores foram encontrados por Jouglard e Brod (2000), em Pelotas, 2,7\%; Mascolli et al. (2002), em Santana de Parnaíba, 15\%; e Fávero et al. (2002), nos Estados de São Paulo, 17,9\%, e Piauí, 19,7\%. Alves et al. (2000), ao examinarem cães domiciliados em Patos, encontraram prevalência muito próxima $(20 \%)$ à observada neste trabalho. Essas diferenças na percentagem de positividade podem ser explicadas pela variedade de fatores que influenciam na ocorrência da leptospirose, com destaque para a topografia, região, temperatura, umidade, precipitação pluviométrica, reservatórios selvagens, reservatórios domésticos e outros fatores ambientais (Alves et al., 2000), bem como pela diferença nas populações caninas estudadas e pela utilização de sorovares distintos no diagnóstico sorológico.

$\mathrm{Na}$ Tab. 1 é apresentada a freqüência de aglutininas anti-Leptospira spp em 285 amostras de soro de cães de Campina Grande, PB, segundo o sorovar e seus respectivos títulos. O sorovar autumnalis foi o mais freqüente, seguido de copenhageni e canicola. Também foram encontradas reações positivas para os sorovares pyrogenes, bratislava, castellonis, icterohaemorrhagiae, australis, andamana e whitcombi. Dados similares foram observados no Brasil por Alves et al. (2000), e no Canadá por Prescott et al. (1999), que também encontraram o sorovar autumnalis como o mais prevalente. A presença do sorovar autumnalis causa preocupação, pois não existe imunidade cruzada entre os diferentes sorovares, e no mercado existem as vacinas compostas, basicamente, pelos sorovares canicola e icterohaemorrhagiae. Isso reforça, ainda mais, a importância da pesquisa continuada no desenvolvimento de novas vacinas contra a leptospirose e a necessidade da inclusão de novos sorovares, visando à elaboração de vacinas mais efetivas e de imunidade mais duradoura. 


\section{Batista et al.}

Tabela 1. Freqüência de aglutininas anti-Leptospira spp em 285 amostras de soro de cães de Campina Grande, PB, segundo o sorovar e seus respectivos títulos

\begin{tabular}{|c|c|c|c|c|c|c|c|}
\hline \multirow{2}{*}{ Sorovar } & \multicolumn{6}{|c|}{ Título } & \multirow{2}{*}{ Total (\%) } \\
\hline & 100 & 200 & 400 & 800 & 1600 & 3200 & \\
\hline castellonis & 2 & 0 & 0 & 0 & 0 & 0 & $2(0,7)$ \\
\hline australis & 0 & 0 & 1 & 0 & 0 & 0 & $1(0,4)$ \\
\hline autumnalis & 11 & 8 & 2 & 0 & 0 & 0 & $21(7,4)$ \\
\hline copenhageni & 4 & 8 & 3 & 1 & 1 & 0 & $17(6)$ \\
\hline pyrogenes & 0 & 1 & 1 & 1 & 1 & 1 & $5(1,8)$ \\
\hline andamana & 1 & 0 & 0 & 0 & 0 & 0 & $1(0,4)$ \\
\hline canicola & 1 & 4 & 0 & 1 & 0 & 0 & $6(2,1)$ \\
\hline whitcombi & 1 & 0 & 0 & 0 & 0 & 0 & $1(0,4)$ \\
\hline icterohaemorrhagiae & 1 & 1 & 0 & 0 & 0 & 0 & $2(0,7)$ \\
\hline bratislava & 0 & 4 & 1 & 0 & 0 & 0 & $5(1,8)$ \\
\hline
\end{tabular}

A prevalência do sorovar copenhageni aponta a importância da população de roedores na transmissão da doença e reforça a necessidade de programas de controle de roedores, adotando, além das medidas ofensivas (desratização), normalmente as únicas utilizadas, a inclusão de modificações ambientais como medidas preventivas (anti-ratização) e a educação em saúde. Os ratos e, em especial, as ratazanas, tidos como os principais portadores universais das leptospiras, são considerados um dos principais responsáveis pela transmissão da doença ao homem (Mascolli et al., 2002).

O sorovar canicola, apontado como o terceiro mais prevalente, alerta para o fato de o cão se constituir em importante fonte de infecção da doença para o homem. O cão é o principal hospedeiro desse sorovar, que apresenta adaptação ao tecido renal canino, podendo ser eliminado pelo portador por longo tempo. O sorovar canicola é citado como o mais encontrado em cães (Furtado et al., 1997).

Sakata et al. (1992) relataram que os sorovares isolados de pacientes humanos hospitalizados com leptospirose no estado de São Paulo, entre 1986 e 1989, foram copenhageni, o mais prevalente $(77,8 \%)$, e canicola $(11,1 \%)$. Esses resultados, como no presente estudo, sugerem que as variantes que circulam entre a população de humanos e cães são as mesmas, uma vez que sua proximidade leva à exposição aos mesmos fatores de risco. Entretanto, a maioria dos trabalhos realizados em cães no Brasil e no exterior assinalam predomínio das variantes icterohaemorrhagiae e canicola ao invés do sorovar copenhageni (Scanziani et al., 1994).
Observou-se a ocorrência de reações sorológicas para sorovares considerados acidentais para cães, como australis e pyrogenes. Segundo Bolin (1996), em determinadas regiões, diferentes sorovares de leptospiras são prevalentes e são associados a um ou mais hospedeiros mantenedores que servem de reservatórios de infecção. Os hospedeiros mantenedores são freqüentemente espécies silvestres e, algumas vezes, animais domésticos e de produção. A transmissão entre hospedeiros mantenedores é eficiente, e a incidência da infecção é relativamente alta. $\mathrm{O}$ contato com os hospedeiros mantenedores ou áreas contaminadas com urina desses pode causar infecção em outras espécies.

A ocorrência de coaglutinação sorológica, em que mais de uma variante apresentou titulação para um mesmo soro, constitui forte indicativo de reações cruzadas. De acordo com os parâmetros adotados no estudo, o sorovar com título mais elevado foi considerado o agente infectante mais provável (Bolin, 1996). A ocorrência de coaglutinação foi citada por Rubel et al. (1997) e Brihuega et al. (1995), que também utilizaram apenas os sorovares com maior título aglutinante, devido à alta freqüência com que um mesmo soro foi capaz de aglutinar mais de um sorovar.

A distribuição do número de cães e a soropositividade para a leptospirose, segundo as variáveis estudadas e os respectivos valores de odds ratio, intervalo de confiança de $95 \%$ (IC 95\%) e a probabilidade de ocorrência ao acaso $(P)$ são apresentadas na Tab. 2. As variáveis selecionadas e oferecidas para a regressão logística multivariada foram idade $(P=0,009)$, 
raça $(P=0,009)$, ocorrência de enchentes $(P=0,062)$ e contato com açude $(P=0,045)$. O modelo final da regressão logística (Tab. 3) apontou como significativas a idade superior a um ano (odds ratio $=3,00$ e $P=0,006)$, a não definição da raça (odds ratio $=4,02$ e $P=0,011$ ) e a ocorrência de enchentes (odds ratio $=2,32$ e $P=0,039)$.

Tabela 2. Distribuição do número de cães e soropositividade para a leptospirose segundo as variáveis estudadas e respectivos valores de odds ratio, intervalo de confiança de 95\% (I.C. 95\%) e probabilidade de ocorrência ao acaso $(P)$

\begin{tabular}{|c|c|c|c|c|}
\hline Variável & No. de cães (\%) & Soropositividade (\%) & Odds ratio (IC 95\%) & $P$ \\
\hline \multicolumn{5}{|l|}{ Idade } \\
\hline Até um ano & $80(28,1)$ & 11,3 & 1 & \\
\hline Superior a um ano & $205(71,9)$ & 25,4 & $2,68(1,25-5,74)$ & 0,009 \\
\hline \multicolumn{5}{|l|}{ Sexo } \\
\hline Macho & $176(61,8)$ & 19,3 & 1 & \\
\hline Fêmea & $109(38,2)$ & 24,8 & $1,38(0,78-2,44)$ & 0,28 \\
\hline \multicolumn{5}{|l|}{ Raça } \\
\hline Definida & $51(17,9)$ & 7,8 & 1 & \\
\hline Indefinida & $234(82,1)$ & 24,4 & $3,78(1,29-15,04)$ & 0,009 \\
\hline \multicolumn{5}{|l|}{ Presença de roedores } \\
\hline Sim & $161(56,5)$ & 21,1 & 1 & \\
\hline Não & $124(43,5)$ & 21,8 & $1,04(0,57-1,91)$ & 0,89 \\
\hline \multicolumn{5}{|c|}{ Ocorrência de enchentes } \\
\hline Não & $249(87,4)$ & 19,7 & 1 & \\
\hline Sim & $36(12,6)$ & 33,3 & $2,04(0,95-4,36)$ & 0,062 \\
\hline \multicolumn{5}{|l|}{ Manejo } \\
\hline Domiciliar & $215(75,4)$ & 20 & 1 & \\
\hline Solto & $70(24,6)$ & 25,7 & $1,39(0,74-2,60)$ & 0,31 \\
\hline \multicolumn{5}{|l|}{ Vacinação } \\
\hline Sim & $33(11,6)$ & 21,2 & 1 & \\
\hline Não & $252(88,4)$ & 21,4 & $1,01(0,39-2,72)$ & 0,98 \\
\hline \multicolumn{5}{|l|}{ Contato com açude } \\
\hline Não & $261(91,6)$ & 19,9 & 1 & \\
\hline Sim & $24(8,4)$ & 37,5 & $2,41(0,91-6,27)$ & 0,045 \\
\hline
\end{tabular}

Tabela 3. Valores da odds ratio, intervalo de confiança de 95\% (IC 95\%) e probabilidade de ocorrência ao acaso $(P)$ estimados pelo método da regressão logística multivariada, segundo as variáveis que permaneceram no modelo final

\begin{tabular}{lcc}
\hline Variável & Odds ratio (IC 95\%) & $P$ \\
\hline Idade & 1 & 0,006 \\
$\quad$ Até um ano & $3,00(1,37-6,53)$ & \\
$\quad$ Superior a um ano & 1 & 0,011 \\
Raça & $4,02(1,37-11,78)$ & \\
$\quad$ Definida & 1 & 0,039 \\
$\quad$ Indefinida & $2,32(1,04-5,16)$ & \\
Ocorrência de enchentes & & \\
Não & & \\
Sim &
\end{tabular}


A regressão logística multivariada destacou a idade superior a um ano, a não definição da raça e a ocorrência de enchentes como fatores de risco para a leptospirose canina. A primeira variável também foi citada por Rubel et al. (1997) e Mascolli et al. (2002), que verificaram soroprevalência significativamente maior em animais com mais de um ano de idade, e por Ward et al. (2002), que verificaram risco significativamente maior em tornarem-se soropositivos cães com idade entre quatro e 10 anos. Isso pode ser explicado pelo tempo de exposição à doença, uma vez que animais mais velhos tiveram mais tempo para entrar em contato com o agente etiológico. Além disso, filhotes normalmente são alvo de cuidados maiores por parte dos proprietários, permanecendo em ambientes mais protegidos, inclusive durante a noite, o que diminui seu contato com possíveis fontes de infecção (Côrtes, 1993). O segundo fator de risco destacado pela análise multivariada, a não definição da raça, também foi citado por Ward et al. (2002). Animais sem raça definida, geralmente, têm mais acesso à rua, o que aumenta o risco de exposição às leptospiras. A ocorrência de enchentes como fator de risco para a leptospirose era esperada, pois durante as épocas chuvosas ocorre a disseminação do agente, eliminado pela urina de roedores e outras fontes de infecção, expondo os animais e o homem ao risco da infecção. Mascolli et al. (2002) não observaram correlação entre a ocorrência de enchentes e a soropositividade para a leptospirose canina em Santana de Parnaíba, justificado pelo fato de o município possuir poucas áreas de alagamento. Neste trabalho, dos 410 proprietários entrevistados, apenas 16 confirmaram a ocorrência de enchentes, o que resultou em número insuficiente para a análise de correlação.

Com relação aos outros parâmetros analisados como fatores de risco, não se confirmou associação estatística com variáveis que normalmente estão relacionadas à ocorrência da doença, como o tipo de confinamento do animal e a presença de roedores. Essa ausência de associação pode ser justificada pelo fato de que as respostas obtidas dos proprietários não são totalmente confiáveis, uma vez que as pessoas podem esquecer ou mesmo ocultar fatos que consideram indesejáveis (como presença de roedores em casa), o que torna muitas vezes inconclusivas as informações obtidas nos questionários.

\section{CONCLUSÕES}

A população canina de Campina Grande está exposta a vários sorovares de Leptospira spp, com maiores preocupações em cães com idade superior a um ano, sem raça definida e provenientes de áreas de enchentes. Há necessidade da elaboração de programas de controle da doença com ações direcionadas para o uso de vacinas homólogas específicas, saneamento do meio ambiente, visando, principalmente, ao controle de roedores e à educação em saúde.

\section{AGRADECIMENTOS}

Os autores agradecem ao $\mathrm{CNPq}$ pela concessão de bolsa de iniciação científica à Carolina de Sousa Américo Batista.

\section{REFERÊNCIAS BIBLIOGRÁFICAS}

ALVES, C.J.; ANDRADE, J.S.L.; VASCONCELLOS, S.A. et al. Avaliação dos níveis de aglutininas anti-leptospira em cães no município de Patos-PB, Brasil. Rev. Bras. Ciênc. Vet., v.7, p.17-21, 2000.

ÁVILA, M.O; FURTADO, L.R.I.; TEXEIRA, M.M. et al. Aglutininas anti-leptospira em cães na área de influência do Centro de Controle de Zoonoses, Pelotas, RS, Brasil, no ano de 1995. Ciênc. Rural, v.28, p.107-110, 1998.

BOLIN, C.A. Diagnosis of leptospirosis: a reemerging disease of companion animals. Semin. Vet. Med. Surg. (Small Anim.), v.11, p.166-171, 1996.

BRASIL. Instituto Brasileiro de Geografia e Estatística. Sistema IBGE de Recuperação Automática - SIDRA. 2003. Disponível em: $<$ http://www.sidra.ibge.gov.br/bda/ $>$. Acessado em: 12 jan. 2003.

BRIHUEGA, B.; HUTTER, E.; LABALLEN, H. Leptospirosis: estúdio serologico em caninos de la ciudad de Rosário. Vet. Argent., v.12, p.720724, 1995. 
CÔRTES, J.A. Aspectos epidemiológicos e ecológicos da leptospirose. In: ENCONTRO NACIONAL EM LEPTOSPIROSE, 3., 1993, Rio de Janeiro. Anais... Rio de Janeiro, 1993. p.53-57.

FAINE, S.; ADLER, B.; BOLIN, C. et al. Leptospira and leptospirosis. 3.ed. Melbourne: MediSei, 1999. 272p.

FÁVERO, A.C.M.; PINHEIRO, S.R.; VASCONCELLOS, S.A. et al. Most frequent serovars of leptospires in serological tests of buffaloes, sheeps, goats, horses, swines and dogs from several brazilian states. Cienc. Rural, v.32, p.613-619, 2002.

FURTADO, L.R.I.; AVILA, M.O.; FEHLBERG, M.F.B. et al. Prevalência e avaliação de fatores de risco à leptospirose canina, no município de Pelotas-RS. Arq. Inst. Biol., v.64, p.57-61, 1997.

GALTON, M.M.; SULZER, C.R.; SANTA ROSA, C.A. et al. Aplication of a microtecnique to the aglutination test for leptospiral antibodies. Appl. Microbiol., v.13, p.81-85, 1965.

HOSMER JR., D.W.; LEMESHOW, S. Applied logistic regression. New York: John Wiley, 1989. 308p.

JOUGLARD, S.D.D.; BROD, C.S. Leptospirose em cães: prevalência e fatores de risco no meio rural do município de Pelotas, RS. Arq. Inst. Biol., v.67, p.181-185, 2000.

MASCOLLI, R.; PINHEIRO, S.R.; VASCONCELLOS, S.A. et al. Inquérito sorológico para leptospirose em cães do Município de Santana de Parnaíba, São Paulo, utilizando a campanha de vacinação anti-rábica do ano de 1999. Arq. Inst. Biol, v.69, p.25-32, 2002.

PRESCOTT, J.F.; KEY, D.; OSUCH, M. Leptospirosis in dogs. Can. Vet. J., v.40, p.430431, 1999.

REICHMANN. M.L.A.B.; PINTO, H.B.F.; NUNES, V.F.P. Vacinação contra a raiva de cães e gatos. São Paulo: Instituto Pasteur, 1999. 32p. (Manuais, 3).

RUBEL, D.; SEIJO, A.; CERNIGOI, B. et al. Leptospira interrogans en una población canina del Gran Buenos Aires: variables asociadas com la seropositividad. Rev. Panam. Salud Publ., v.2, p.102-105, 1997.

SAKATA, E.E.; YASUDA, P.H.; ROMERO, E.C. et al. Sorovares de Leptospira interrogans isoladas de casos de leptospirose humana em São Paulo, Brasil. Rev. Inst. Med. Trop. S. Paulo, v.34, p.217-221, 1992.

SCANZIANI, E.; CALCATERRA, S.; TAGLIABUE, S. Serologic findings in cases of acute leptospirosis in the dog. J. Small Anim. Pract., v.35, p.257-260, 1994.

VASCONCELLOS, S.A. Leptospirose animal. In: ENCONTRO NACIONAL EM LEPTOSPIROSE, 3., 1993, Rio de Janeiro. Anais... Rio de Janeiro, 1993. p.62-65.

WARD, M.P.; GLICKMAN, L.T.; GUPTILL, L.F. Prevalence of and risk factors for leptospirosis among dogs in the United States and Canada: 677 cases (1970-1998). J. Am. Vet. Med. Assoc., v.220, p.53-58, 2002. 